\title{
Linear Quadratic Nash Differential Games of Stochastic Singular Systems
}

\author{
Haiying ZHOU \\ School of Management, Guangdong University of Technology, Guangzhou 510520, China \\ E-mail: cindychou@163.com \\ Huainian ZHU \\ School of Management, Guangdong University of Technology, Guangzhou 510520, China \\ Chengke ZHANG \\ School of Economics and Commerce, Guangdong University of Technology, Guangzhou 510520, China
}

\begin{abstract}
In this paper, we deal with the Nash differential games of stochastic singular systems governed by Itô-type equation in finite-time horizon and infinite-time horizon, respectively. Firstly, the Nash differential game problem of stochastic singular systems in finite time horizon is formulated. By applying the results of stochastic optimal control problem, the existence condition of the Nash strategy is presented by means of a set of cross-coupled Riccati differential equations. Similarly, under the assumption of the admissibility of the stochastic singular systems, the existence condition of the Nash strategy in infinite-time horizon is presented by means of a set of cross-coupled Riccati algebraic equations. The results show that the strategies of each players interact.
\end{abstract}

Keywords stochastic singular systems; Nash differential games; finite-time horizon; infinite-time horizon

\section{Introduction}

Singular systems, also known as descriptor systems, generalized state-space systems and implicit systems are described by differential-algebraic equations. Singular systems have been extensively studied over the past decades due to the fact that they can describe a great many natural phenomena in physical systems such as microelectronic circuits, economics, demography and so on ${ }^{[1-4]}$.

As is well known, environmental noise exists and cannot be neglected in many dynamical systems $^{[5-9]}$. And there are some results about the research of LQ control for stochastic singular systems: Balasubramaniam and Kumaresan ${ }^{[10]}$ discussed a solution of generalized matrix Riccati differential equation for indefinite stochastic linear quadratic singular system using neural networks. The optimal control problem for a class of stochastic LQ singular periodic neuro Takagi-Sugeno fuzzy systems by using ant colony programming was studied in [11]. Kumaresan

Received January 21, 2014, accepted October 15, 2014

Supported by National Natural Science Foundation of China (71171061); Natural Science Foundation of Guangdong Province (S2011010004970); China Postdoctoral Science Foundation (2014M552177) 
and Kuru ${ }^{[12]}$ investigated the optimal control problem for stochastic linear singular TakagiSugeno fuzzy delay systems with quadratic performance using genetic programming. Zhang and Xing ${ }^{[13]}$ investigated the stability and optimal control for stochastic singular systems.

On the other hand, during the past decades, differential games have been playing a central role in economy, ecology and elsewhere, such as optimizing behavior and enduring consequences of decisions. For that reason, this framework has developed into a major research field in control theory, and has many applications for solving real world problems, see [14-16]. The differential games of singular systems can describe many practical situations and have been widely studied by many researchers for several decades. Among these, most researchers studied the differential games of deterministic singular systems in finite-time horizon and infinite-time horizon, we refer the reader to [17-21] and the references therein.

However, to the best of our knowledge, few results have been obtained of the differential games for stochastic singular systems. Motivated by this, we deal with the LQ Nash differential games of stochastic singular systems.

It is worth mentioning that many researchers discussed the Nash differential games of singular systems by disintegrating the systems into two: fast and slow ${ }^{[18,19,21]}$. Recently Zhang and Xing deal with the stochastic singular systems as a whole by using the square completion technique, and get the results for optimal control problems involving new stochastic generalized Riccati equation. Inspired by this, we apply the same method to the corresponding differential game problems and get a set of cross-coupled stochastic Riccati equations. Actually, the conclusion is more intuitive and calculation is more easily.

The rest of the paper is organized as follows: Some important definitions and lemmas which are useful to obtain the main results are introduced in section 2 . The differential games of stochastic singular system in finite-time horizon are discussed in section 3. In this case, the problem is formulated firstly. Then, the corresponding cross-coupled Riccati differential equations are derived and the existence of their solutions is shown to be sufficient conditions for the Nash strategies. The differential games of stochastic singular system in infinite-time horizon discussed in section 4. Similarly, the corresponding cross-coupled Riccati algebraic equations are derived and the existence of their solutions is shown to be sufficient conditions for the Nash strategies. In section 5 , the conclusions are given.

For convenience, we will make use of the following notations throughout this paper:

$A^{\prime}$ : transpose of a matrix or vector $A ; A^{-1}$ : inverse of a matrix or vector $A ; A>0(A \geq 0)$ : positive definite (positive semi-definite) matrix $A ; \varepsilon[\cdot]$ : the expectation operator with respect to the given probability measure $P . R^{n}$ : the $n$-dimensional Euclidean space; $S^{n \times n}$ : the set of all $n \times n$ matrices; $S^{n}$ : the space of all $n \times n$ symmetric matrices; $S_{+}^{n}$ : the space of all nonnegative definite matrices of $S^{n} ; \operatorname{rank}(A)$ : the rank of $A ; \operatorname{deg}(\operatorname{det}(s I-A))$ : degree of determinant $s I$ $-A$.

\section{Preliminaries}

In this section, we introduce some important definitions and lemmas which are useful to obtain our main results later.

Throughout this paper, let $\left(\Omega, F,\left\{F_{t}\right\}_{t \geq 0}, P\right)$ be a given filtered probability space where 
there exists a standard one dimensional Wiener process $\{w(t)\}_{t \geq 0}$. Consider the following stochastic singular systems

$$
\left\{\begin{array}{l}
E \mathrm{~d} x(t)=A x(t) \mathrm{d} t+C x(t) \mathrm{d} W(t) \\
x(0)=x_{0}, t \geq 0
\end{array}\right.
$$

where $x_{0} \in R^{n}$ is the initial state of the systems, $A \in R^{n \times n}, C \in R^{n \times n}$ are known coefficient matrices associated with $x(t)$, respectively. $E$ is a known singular matrix with $\operatorname{rank}(E)=r \leq n$.

Assumption $1^{[22]}$ There is a pair of nonsingular matrices $P \in R^{n \times n}$ and $Q \in R^{n \times n}$ for the triplet $(E, A, C)$ such that the following conditions are satisfied:

$$
P E Q=\left[\begin{array}{cc}
I_{r} & 0 \\
0 & 0
\end{array}\right], \quad P A Q=\left[\begin{array}{cc}
A_{1} & B_{1} \\
0 & D_{1}
\end{array}\right], \quad P C Q=\left[\begin{array}{cc}
C_{1} & 0 \\
0 & I_{n-r}
\end{array}\right],
$$

where $A_{1} \in R^{n \times n}, B_{1} \in R^{r \times(n-r)}, C_{1} \in R^{n \times n}, D_{1} \in R^{(n-r) \times(n-r)}$.

Hence, we have the following result for the existence and uniqueness of solution to the system (1).

Lemma $\mathbf{1}^{[13]}$ System (1) has a unique solution if the above assumption holds.

Definition $1^{[23]}$

(I) The system (1) is said to be regular if $\operatorname{det}(s E-A)$ is not identically zero;

(II) The system (1) is said to be impulse-free if $\operatorname{deg}(\operatorname{det}(s E-A))=\operatorname{rank}(E)$;

(III) The system (1) is said to be mean-square stable if $\lim _{t \rightarrow \infty} \varepsilon|x(t)|^{2}=0$;

(IV) The system (1) is said to be mean-square admissible, if the system is regular, impulse-free and mean-square stable.

Lemma $2^{[13]}$ System (1) is mean-square admissible if there exists a nonsingular matrix $G$, such that the following coupled LMIs hold:

$$
\begin{aligned}
& E^{\prime} G=G^{\prime} E \geq 0 \\
& A^{\prime} G+G^{\prime} A+C^{\prime} E^{\prime} G C<0
\end{aligned}
$$

Next, the Nash differential games for stochastic singular systems in finite-time horizon and infinite-time horizon are discussed, respectively.

\section{Stochastic Nash differential games in finite-time horizon}

\subsection{Problem formulation}

We consider the following systems

$$
\left\{\begin{array}{l}
E \mathrm{~d} x(t)=\left[A x(t)+B_{1} u_{1}(t)+B_{2} u_{2}(t)\right] \mathrm{d} t+C x(t) \mathrm{d} w(t) \\
x(0)=x_{0}, t \in[0, T]
\end{array}\right.
$$

The quadratic cost function associated with each player is

$$
\begin{aligned}
& J_{\tau}\left(u_{1}, u_{2} ; x_{0}, 0\right) \\
& =\varepsilon\left\{\frac{1}{2} \int_{0}^{T}\left[x^{\prime}(t) Q_{\tau} x(t)+u_{1}^{\prime}(t) R_{\tau 1} u_{1}(t)+u_{2}^{\prime}(t) R_{\tau 2} u_{2}(t)\right] \mathrm{d} t+\frac{1}{2} x^{\prime}(T) H_{\tau} x(T)\right\}, \tau=1,2
\end{aligned}
$$


where $x(t) \in R^{n}$ is the system state, the coefficient matrices $A, B_{1}, B_{2}, C$ are assumed to be real constant matrices with appropriate dimensions. $E$ is a known singular matrix with $\operatorname{rank}(E)$ $=r \leq n, u_{1}(t) \in R^{m}$ and $u_{2}(t) \in R^{l}$ represent the two players control inputs respectively. The matrices $Q_{\tau}, H_{\tau}(\tau=1,2)$ are assumed to be positive semi-definite symmetric matrices with appropriate dimensions, $R_{\tau 1}$ and $R_{\tau 2}(\tau=1,2)$ are assumed to be positive definite symmetric matrices with appropriate dimensions.

The strategies of the two players are denoted by $u_{1}(t)$ and $u_{2}(t)$, which belong to strategy spaces $\Gamma_{1}$ and $\Gamma_{2}$, respectively. In this note, the restriction that $\Gamma_{1}$ and $\Gamma_{2}$ are composed of linear feedback strategies of the form, i.e.

$$
u_{1}(t)=K_{1}(t) x(t), u_{2}(t)=K_{2}(t) x(t)
$$

Definition 2 A linear feedback strategy pair $\left(u_{1}(t), u_{2}(t)\right) \in \Gamma_{1}^{a} \times \Gamma_{2}^{a} \subset \Gamma_{1} \times \Gamma_{2}$ is called an admissible strategy pair if the closed-loop system obtained has no impulsive solution. Correspondingly, $\Gamma_{1}^{a} \times \Gamma_{2}^{a} \subset \Gamma_{1} \times \Gamma_{2}$ is called the admissible strategy space.

Definition 3 An admissible linear feedback strategy pair $\left(u_{1}^{*}(t), u_{2}^{*}(t)\right) \in \Gamma_{1}^{a} \times \Gamma_{2}^{a}$ constitutes a Nash equilibrium pair if

$$
J_{1}\left(u_{1}^{*}, u_{2}^{*} ; x_{0}, 0\right) \leq J_{1}\left(u_{1}, u_{2}^{*} ; x_{0}, 0\right), J_{2}\left(u_{1}^{*}, u_{2}^{*} ; x_{0}, 0\right) \leq J_{2}\left(u_{1}^{*}, u_{2} ; x_{0}, 0\right)
$$

for all $\left(u_{1}^{*}(t), u_{2}^{*}(t)\right) \in \Gamma_{1}^{a} \times \Gamma_{2}^{a},\left(u_{1}(t), u_{2}^{*}(t)\right) \in \Gamma_{1}^{a} \times \Gamma_{2}^{a},\left(u_{1}^{*}(t), u_{2}(t)\right) \in \Gamma_{1}^{a} \times \Gamma_{2}^{a}$.

\subsection{One-player case}

First, a one-player case is discussed. The result obtained for that particular case is used as the basis for the derivation of the results for the 2-player case.

Consider linear quadratic (LQ) stochastic controlled singular systems in the following form.

$$
\begin{aligned}
& \min _{u} J\left(u ; x_{0}, 0\right)=\varepsilon\left\{\frac{1}{2} \int_{0}^{T}\left[x^{\prime}(t) Q x(t)+u^{\prime}(t) R u(t)\right] \mathrm{d} t+\frac{1}{2} X^{\prime}(T) H X(T)\right\} \\
& \text { s.t. } \\
& \left\{\begin{array}{l}
E \mathrm{~d} x(t)=[A x(t)+B u(t)] \mathrm{d} t+[C x(t)+D u(t)] \mathrm{d} w(t) \\
x(0)=x_{0}
\end{array}\right.
\end{aligned}
$$

Theorem $1^{[13]}$ If there exists a solution $P(t) \in S_{+}^{n}$ for the following Riccati differential equations

$$
\left\{\begin{array}{l}
E^{\prime} \dot{P}(t) E+E^{\prime} P(t) A+A^{\prime} P(t) E+C^{\prime} E^{\prime} P(t) E C \\
+K^{\prime}(t)\left(B^{\prime} P(t) E+D^{\prime} E^{\prime} P(t) E C\right)+Q=0 \\
E^{\prime} P(T) E=H \\
K(t)=-\left(R+D^{\prime} E^{\prime} P(t) E D\right)^{-1}\left(B^{\prime} P(t) E+D^{\prime} E^{\prime} P(t) E C\right) \\
R+D^{\prime} E^{\prime} P(t) E D>0
\end{array}\right.
$$

Then, the linear state feedback optimal control law for the $L Q$ problem is

$$
u^{*}(t)=K(t) x(t)=-\left(R+D^{\prime} E^{\prime} P(t) E D\right)^{-1}\left(B^{\prime} P(t) E+D^{\prime} E^{\prime} P(t) E C\right) x(t)
$$

Moreover, the corresponding function is

$$
J\left(u^{*} ; x_{0}, 0\right)=\frac{1}{2} x_{0}^{\prime} E^{\prime} P(0) E x_{0}
$$




\subsection{Main result}

The solution for the finite-time horizon stochastic Nash differential games is given below.

Theorem 2 For system (3) (4), assume that for any $\left(u_{1}(t), u_{2}(t)\right) \in \Gamma_{1}^{a} \times \Gamma_{2}^{a}$, the following stochastic generalized Riccati differential equations (11) and (12) admit the solutions $\left(P_{1}(t), P_{2}(t)\right) \in S_{+}^{n} \times S_{+}^{n}:$

$$
\begin{aligned}
& \left\{\begin{array}{l}
E^{\prime} \dot{P}_{1}(t) E+E^{\prime} P_{1}(t)\left(A+B_{2} K_{2}(t)\right)+\left(A+B_{2} K_{2}(t)\right)^{\prime} P_{1}(t) E+Q_{1} \\
+C^{\prime} E^{\prime} P_{1}(t) E C+K_{2}^{\prime}(t) R_{12} K_{2}(t)+K_{1}^{\prime}(t) B_{1}^{\prime} P_{1}(t) E=0 \\
E^{\prime} P_{1}(T) E=H_{1} \\
K_{1}(t)=-R_{11}^{-1} B_{1}^{\prime} P_{1}(t) E
\end{array}\right. \\
& \left\{\begin{array}{l}
E^{\prime} \dot{P}_{2}(t) E+E^{\prime} P_{2}(t)\left(A+B_{1} K_{1}(t)\right)+\left(A+B_{1} K_{1}(t)\right)^{\prime} P_{2}(t) E+Q_{2} \\
+C^{\prime} E^{\prime} P_{2}(t) E C+K_{1}^{\prime}(t) R_{21} K_{1}(t)+K_{2}^{\prime}(t) B_{2}^{\prime} P_{2}(t) E=0 \\
E^{\prime} P_{2}(T) E=H_{2} \\
K_{2}(t)=-R_{22}^{-1} B_{2}^{\prime} P_{2}(t) E
\end{array}\right.
\end{aligned}
$$

If the system (3) is mean-square admissible, then

i) The problem of finite-time horizon stochastic Nash differential games admits a pair of solutions $\left(u_{1}^{*}(t), u_{2}^{*}(t)\right)$ with

$$
u_{1}^{*}(t)=K_{1}(t) x(t), u_{2}^{*}(t)=K_{2}(t) x(t)
$$

ii) The optimal cost functions incurred by playing strategies $\left(u_{1}^{*}(t), u_{2}^{*}(t)\right)$ are

$$
J_{\tau}\left(u_{1}^{*}, u_{2}^{*} ; x_{0}, 0\right)=\frac{1}{2} x_{0}^{\prime} P_{\tau}(0) x_{0},(\tau=1,2)
$$

Proof Now, let us consider the following LQ problem in which the cost function (15) is minimal at $K_{i}(t)=K_{i}^{*}(t)$

$$
\begin{aligned}
& J_{i}\left(u_{i}, u_{j}^{*} ; x_{0}, 0\right) \\
& =\varepsilon\left\{\frac{1}{2} \int_{0}^{T}\left[x^{\prime}(t)\left(Q_{i}+K_{j}^{*^{\prime}}(t) R_{i j} K_{j}^{*}(t)+K_{i}^{\prime}(t) R_{i i} K_{i}(t)\right) x(t)\right] \mathrm{d} t+\frac{1}{2} x^{\prime}(T) H_{i} x(T)\right\}
\end{aligned}
$$

where $x(t)$ follows from

$$
\left\{\begin{array}{l}
E \mathrm{~d} x(t)=\left[A+B_{j} K_{j}^{*}(t)+B_{i} K_{i}(t)\right] x(t) \mathrm{d} t+C x(t) \mathrm{d} w(t) \\
x(0)=x_{0}, i, j=1,2, i \neq j
\end{array}\right.
$$

Note that the LQ problem $(15) \sim(16)$ coincides with the LQ problem $(3) \sim(4)$ in Theorem 1. Applying Theorem 1 to the LQ problem $(15) \sim(16)$ as

$$
\begin{aligned}
& A+B_{j} K_{j}^{*}(t) \Rightarrow A, B_{i} \Rightarrow B, C \Rightarrow C, 0 \Rightarrow D, \\
& Q_{i}+K_{j}^{*^{\prime}}(t) R_{i j} K_{j}^{*}(t) \Rightarrow Q, R_{i i} \Rightarrow R, H_{i} \Rightarrow H .
\end{aligned}
$$

Note that $R_{i i}>0$, yields the fact that the function $J_{i}\left(u_{i}, u_{j}^{*} ; x_{0}, 0\right)$ is minimal at $u^{*}(t)=-\left(R+D^{\prime} E^{\prime} P(t) E D\right)^{-1}\left(B^{\prime} P(t) E+D^{\prime} E^{\prime} P(t) E C\right) x(t) \Rightarrow u_{i}^{*}(t)=-R_{i i}^{-1} B_{i}^{\prime} P_{i}(t) E x(t)$ 
Moreover, the optimal value is $\frac{1}{2} x_{0}^{\prime} E^{\prime} P_{i}(0) E x_{0}$.

So this completes the proof.

\section{Stochastic Nash differential games in infinite-time horizon}

\subsection{Preliminaries}

Firstly, let us recall the stability of the infinite-time stochastic singular system.

Consider the following system

$$
\left\{\begin{array}{l}
E \mathrm{~d} x(t)=[A x(t)+B u(t)] \mathrm{d} t+C x(t) \mathrm{d} w(t) \\
x(0)=x_{0}, t \geq 0
\end{array}\right.
$$

where the coefficient matrices $A, B, C$ are real constant matrices with appropriate dimensions, and a process $u(\cdot) \in R^{m}$ is the control input.

For the system (18), we consider the following state feedback controller

$$
u(t)=\hat{K} x(t)
$$

where $\hat{K}$ is a constant matrix of appropriate dimensions, to be determined.

Furthermore, we get the corresponding closed-loop system

$$
\left\{\begin{array}{l}
E \mathrm{~d} x(t)=(A+B \hat{K}) x(t) \mathrm{d} t+C x(t) \mathrm{d} w(t) \\
x(0)=x_{0} \in R^{n}, t \geq 0
\end{array}\right.
$$

Definition $4^{[13]}$ The system (18) is called mean-square stabilizable if there exists a state feedback control law (19) such that the closed-loop system (20) is mean-square stable.

\subsection{Problem formulation}

Consider the following stochastic singular systems

$$
\left\{\begin{array}{l}
E \mathrm{~d} x(t)=\left[A x(t)+B_{1} u_{1}(t)+B_{2} u_{2}(t)\right] \mathrm{d} t+C x(t) \mathrm{d} w(t) \\
x(0)=x_{0}, t \geq 0
\end{array}\right.
$$

where $x(t) \in R^{n}$ is the system state, the coefficient matrices $A, C, B_{1}, B_{2}$ are real constant matrices with appropriate dimensions. $E$ is a known singular matrix with $\operatorname{rank}(E)=r \leq n$.

The quadratic cost functions associated with each player are

$$
\begin{aligned}
& J_{\tau}\left(u_{1}, u_{2} ; x_{0}, 0\right) \\
& =\varepsilon\left\{\frac{1}{2} \int_{0}^{\infty}\left[x^{\prime}(t) Q_{\tau} x(t)+u_{1}^{\prime}(t) R_{\tau 1} u_{1}(t)+u_{2}^{\prime}(t) R_{\tau 2} u_{2}(t)\right] \mathrm{d} t\right\}, \tau=1,2
\end{aligned}
$$

where the weighting matrices $Q_{\tau}(\tau=1,2)$ are given symmetric positive semi-definite matrices, $R_{\tau 1}, R_{\tau 2}(\tau=1,2)$ are given symmetric positive definite matrices.

In this note, the restriction that $\Gamma_{1}$ and $\Gamma_{2}$ are composed of linear feedback strategies of the form, i.e.

$$
u_{1}=K_{1} x(t), u_{2}=K_{2} x(t) .
$$

Therefore, we are looking for an admissible linear feedback strategy pair $\left(u_{1}^{*}, u_{2}^{*}\right) \in \Gamma_{1}^{a} \times \Gamma_{2}^{a}$ that satisfies

$$
J_{1}\left(u_{1}^{*}, u_{2}^{*} ; x_{0}, 0\right) \leq J_{1}\left(u_{1}, u_{2}^{*} ; x_{0}, 0\right), J_{2}\left(u_{1}^{*}, u_{2}^{*} ; x_{0}, 0\right) \leq J_{2}\left(u_{1}^{*}, u_{2} ; x_{0}, 0\right)
$$


for all $\left(u_{1}^{*}, u_{2}^{*}\right) \in \Gamma_{1}^{a} \times \Gamma_{2}^{a},\left(u_{1}, u_{2}^{*}\right) \in \Gamma_{1}^{a} \times \Gamma_{2}^{a},\left(u_{1}^{*}, u_{2}\right) \in \Gamma_{1}^{a} \times \Gamma_{2}^{a}$.

The following basic assumption is imposed throughout this section.

Assumption 2 The system (21) is mean-square stabilizable.

\subsection{Main result}

Theorem 3 For system (21) (22), suppose the following generalized Riccati algebraic equations (24) and (25) admit solutions $\left(P_{1}, P_{2}\right) \in S_{+}^{n} \times S_{+}^{n}$ :

$$
\begin{aligned}
& \left\{\begin{array}{l}
E^{\prime} P_{1}\left(A+B_{2} K_{2}\right)+\left(A+B_{2} K_{2}\right)^{\prime} P_{1} E+Q_{1} \\
+C^{\prime} E^{\prime} P_{1} E C+K_{2}^{\prime} R_{12} K_{2}+K_{1}^{\prime} B_{1}^{\prime} P_{1} E=0 \\
K_{1}=-R_{11}^{-1} B_{1}^{\prime} P_{1} E
\end{array}\right. \\
& \left\{\begin{array}{l}
E^{\prime} P_{2}\left(A+B_{1} K_{1}\right)+\left(A+B_{1} K_{1}\right)^{\prime} P_{2} E+Q_{2} \\
+C^{\prime} E^{\prime} P_{2} E C+K_{1}^{\prime} R_{21} K_{1}+K_{2}^{\prime} B_{2}^{\prime} P_{2} E=0 \\
K_{2}=-R_{22}^{-1} B_{2}^{\prime} P_{2} E
\end{array}\right.
\end{aligned}
$$

Then

i) The problem of infinite-time horizon stochastic differential games admits a pair of $\left(u_{1}^{*}, u_{2}^{*}\right)$ with

$$
u_{1}^{*}=K_{1} x(t), u_{2}^{*}=K_{2} x(t)
$$

ii) The optimal cost functions incurred by playing strategies $\left(u_{1}^{*}, u_{2}^{*}\right)$ are

$$
J_{\tau}\left(u_{1}^{*}, u_{2}^{*} ; x_{0}, 0\right)=\frac{1}{2} x_{0}^{\prime} P_{\tau} x_{0},(\tau=1,2)
$$

Proof is similar with the corresponding proof in Theorem 3.

\section{Conclusions}

In this paper, we dealt with the stochastic Nash games in finite-time horizon and infinitetime horizon, respectively. Basing on the results of the stochastic optimal control problem, the Nash strategies for stochastic singular systems are derived by the method of a set of crosscoupled stochastic Riccati equations. We note that the strategies of the two players are interacted and it has applications for solving real world problems. For example, considering the investment stock problem of a security company, both the investors and security company need to consider the strategies of each other when they choose the strategies. Hence, it would be necessary to consider the differential games for the stochastic singular systems to obtain an equibrilium. Also the results are easily obtained from those of LQ scochastic singular systems, the method and the stochastic cross-coupled Raccti equations are new for the Nash games of the scochastic singular system. Due to various reasons, we only deal with the open-loop Nash equilibrium for the stochastic singular systems, and the close-loop Nash equilibrium is the next step of research.

\section{References}

[1] Lewis F L. A survey of linear singular systems. Circuits, Systems and Signal Processing, 1986, 5(1): 3-36. 
[2] Brenan K E, Campbell S L V, Petzold L R. Numerical solution of initial-value problems in differentialalgebraic equations. Siam, 1989.

[3] Liu C, Zhang Q, Feng Y, et al. Complex dynamics in a harvested differential-algebraic eco-epidemiological model. International Journal of Information and Systems Sciences, 2009, 5(3-4): 311-324.

[4] Masubuchi I, Kamitane $\mathrm{Y}$, Ohara $\mathrm{A}$, et al. $\mathrm{H}_{\infty}$ control for descriptor systems: A matrix inequalities approach. Automatica, 1997, 33(4): 669-673.

[5] Boukas E K. Stabilization of stochastic singular nonlinear hybrid systems. Nonlinear Analysis: Theory, Methods \& Applications, 2006, 64(2): 217-228.

[6] Gerdin M, Glad T, Ljung L. Well-posedness of filtering problems for stochastic linear DAE models. Decision and Control, 2005 and 2005 European Control Conference. CDC-ECC'05. 44th IEEE Conference on. IEEE, 2005: 350-355.

[7] Gerdin M, Sjoberg J. Nonlinear stochastic differential-algebraic equations with application to particle filtering. Decision and Control, 2006 45th IEEE Conference on. IEEE, 2006: 6630-6635.

[8] Mao X, Yuan C. Stochastic differential equations: With Markovian switching. Imperial College Press, 2006.

[9] Schein O, Denk G. Numerical solution of stochastic differential-algebraic equations with applications to transient noise simulation of microelectronic circuits. Journal of Computational and Applied Mathematics, 1998, 100(1): 77-92.

[10] Balasubramaniam P, Kumaresan N. Solution of generalized matrix Riccati differential equation for indefinite stochastic linear quadratic singular system using neural networks. Applied Mathematics and Computation, 2008, 204(2): 671-679.

[11] Kumaresan N. Optimal control for stochastic linear quadratic singular periodic neuro Takagi-Sugeno (TS) fuzzy system with singular cost using ant colony programming. Applied Mathematical Modelling, 2011, 35(8): 3797-3808.

[12] Kumaresan N, Kuru R. Optimal control for stochastic linear quadratic singular Takagi-Sugeno fuzzy delay system using genetic programming. Applied Soft Computing, 2012, 12(8): 2085-2090.

[13] Zhang Q, Xing S. Stability analysis and optimal control of stochastic singular systems. Optimization Letters, 2013: 1-16.

[14] Basar T, Olsder G J. Dynamic noncooperative game theory[M]. 2nd ed. Philadelphia, PA: SIAM, 1999.

[15] Dockner E, Jorgensen S, Long N V, et al. Differential games in economics and management science. Cambridge University Press, 2000.

[16] Friesz, Terry L. Dynamic optimization and differential games. Springer, 2009.

[17] Xu H, Mizukami K. New sufficient conditions for linear feedback closed-loop Stackelberg strategy of descriptor systems. IEEE Transactions on Automatic Control, 1994, 39(5): 1097-1102.

[18] Engwerda J C. The open-loop linear quadratic differential game for index one descriptor systems. Automatica, 2009, 45(2): 585-592.

[19] Mizukami K, Tetsushi K. On closed-loop Nash equilibrium solutions for continuous descriptor systems. Bulletin of Hiroshima Koukusai Gakuin University, 2001, 34: 51-60.

[20] $\mathrm{Xu} \mathrm{H}$, Mizukami K. On the Isaacs equation of differential games for descriptor systems. Journal of optimization theory and applications, 1994, 83(2): 405-419.

[21] Xu H, Mizukami K. Linear-quadratic zero-sum differential games for generalized state space systems. IEEE Transactions on Automatic Control, 1994, 39(1): 143-147.

[22] Boukas E K. Control of singular systems with random abrupt changes. Springer, Berlin, 2008.

[23] Lu R, Dai X, Du W, et al. Robust $\mathrm{H}_{\infty}$ output feedback control for uncertain stochastic singular systems. 2008 Control and Decision Conference, CCDC 2008, Chinese. IEEE, 2008: 4344-4349. 